\title{
Solitary pulmonary nodule imaging approaches and the role of optical fibre-based technologies
}

\author{
Susan Fernandes $\mathbb{0}^{1}$, Gareth Williams ${ }^{1}$, Elvira Williams ${ }^{1}$, Katjana Ehrlich ${ }^{1}$, \\ James Stone ${ }^{1,2}$, Neil Finlayson (1) 1,3, Mark Bradley ${ }^{1,4}$, Robert R. Thomson ${ }^{1,5}$, \\ Ahsan R. Akram ${ }^{1}$ and Kevin Dhaliwal ${ }^{1}$
}

Affiliations: ${ }^{1}$ Centre for Inflammation Research, Queen's Medical Research Institute, The University of Edinburgh, Edinburgh, UK. ${ }^{2}$ Centre for Photonics and Photonic Materials, Dept of Physics, The University of Bath, Bath, UK. ${ }^{3}$ Institute for Integrated Micro and Nano Systems, School of Engineering. The University of Edinburgh, Edinburgh, UK. "EaStCHEM, School of Chemistry, The University of Edinburgh, Edinburgh, UK. ${ }^{5}$ Institute of Photonics and Quantum Sciences, School of Engineering and Physical Sciences, Heriot-Watt University, Edinburgh, UK.

Correspondence: Susan Fernandes, Centre for Inflammation Research, Queen's Medical Research Institute, The University of Edinburgh, 47 Little France Crescent, Edinburgh, EH16 4TJ, UK. E-mail: susan.fernandesded.ac.uk

@ERSpublications

Solitary pulmonary nodules are a huge diagnostic challenge. Optical fibre-based technologies, in conjunction with bronchoscopic and transthoracic platforms, are promising novel diagnostic tools in the detection of early lung cancer. https://bit.ly/3cOIDx4

Cite this article as: Fernandes S, Williams G, Williams E, et al. Solitary pulmonary nodule imaging approaches and the role of optical fibre-based technologies. Eur Respir J 2021; 57: 2002537 [https://doi.org/ $10.1183 / 13993003.02537-2020]$.

ABSTRACT Solitary pulmonary nodules (SPNs) are a clinical challenge, given there is no single clinical sign or radiological feature that definitively identifies a benign from a malignant SPN. The early detection of lung cancer has a huge impact on survival outcome. Consequently, there is great interest in the prompt diagnosis, and treatment of malignant SPNs. Current diagnostic pathways involve endobronchial/ transthoracic tissue biopsies or radiological surveillance, which can be associated with suboptimal diagnostic yield, healthcare costs and patient anxiety. Cutting-edge technologies are needed to disrupt and improve, existing care pathways. Optical fibre-based techniques, which can be delivered via the working channel of a bronchoscope or via transthoracic needle, may deliver advanced diagnostic capabilities in patients with SPNs. Optical endomicroscopy, an autofluorescence-based imaging technique, demonstrates abnormal alveolar structure in SPNs in vivo. Alternative optical fingerprinting approaches, such as timeresolved fluorescence spectroscopy and fluorescence-lifetime imaging microscopy, have shown promise in discriminating lung cancer from surrounding healthy tissue. Whilst fibre-based Raman spectroscopy has enabled real-time characterisation of SPNs in vivo. Fibre-based technologies have the potential to enable in situ characterisation and real-time microscopic imaging of SPNs, which could aid immediate treatment decisions in patients with SPNs. This review discusses advances in current imaging modalities for evaluating SPNs, including computed tomography (CT) and positron emission tomography-CT. It explores the emergence of optical fibre-based technologies, and discusses their potential role in patients with SPNs and suspected lung cancer.

This article has supplementary material available from erj.ersjournals.com

Received: 25 Nov 2019 | Accepted: 29 Sept 2020

Copyright $\odot$ ERS 2021. This article is open access and distributed under the terms of the Creative Commons Attribution Licence 4.0. 


\section{Introduction}

Solitary pulmonary nodules (SPNs) are defined as spherical radiographic opacities, measuring less than $3 \mathrm{~cm}$ in diameter, which are surrounded by aerated lung and are not associated with other thoracic abnormalities [1]. They are further sub-classified as solid, part-solid and ground-glass nodules based upon computed tomography (CT) attenuation (figure 1).

The widespread use of CT in clinical practice has made it commonplace to detect nodules incidentally, with a prevalence of $13 \%$ in non-screening populations [1]. In screening populations, CT can detect nodules in $\sim 50 \%$ of individuals aged over 50 years with a smoking history [2]. SPNs remain an evolving clinical challenge, which cause clinical and diagnostic uncertainty. Whilst the majority will be benign, some will represent early treatable lung cancer. Lung cancer remains the most common malignancy and the most common cause of cancer related deaths worldwide [3]. Early detection has an impact on survival outcome: the 5-year survival rate in stage I lung cancer is $80 \%$, compared with $10 \%$ in stage IV disease [4]. Consequently, there is great interest in the early identification and treatment of malignant SPNs.

Advances in low-dose CT (LDCT) technology have enabled numerous lung cancer screening studies. Collectively, these studies demonstrate a mean nodule prevalence of 33\% (17-53\%) with a lung cancer prevalence of $1.4 \%(0.5-2.7 \%)$ [1]. The largest screening study, the National Lung Screening Trial, demonstrated a $20 \%$ relative reduction in lung cancer mortality with LDCT [5]. The NELSON study has recently shown that LDCT screening led to $24 \%$ reduction in 10 -year lung cancer mortality in high risk men [6]. The future implementation of national lung cancer screening initiatives [7, 8] will generate increasing numbers of secondary care referrals with SPNs requiring further evaluation.

There is no single clinical sign or radiological feature that definitively identifies malignant SPNs. The British Thoracic Society (BTS) guidelines advocate the use of validated risk prediction models [9, 10], coupled with radiological assessment of nodule size and morphology, to characterise the risk of malignancy [1]. The majority of subcentimetre SPNs undergo radiological surveillance, observing for evidence of growth or the development of sinister features. This results in a significant number of "possible lung cancers" with associated radiation exposure, healthcare costs [11] and patient anxiety [12].

In high-risk SPNs, pre-operative histological confirmation is recommended $[1,13]$. Recent advances in bronchoscopic and CT-guided transthoracic approaches mean it is possible to reach the majority of SPNs. Traditionally, CT-guided transthoracic needle biopsy (CT-TNB) has been the preferred approach due to an

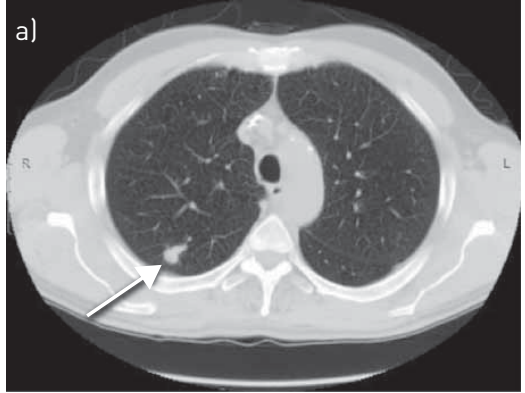

Solid nodule

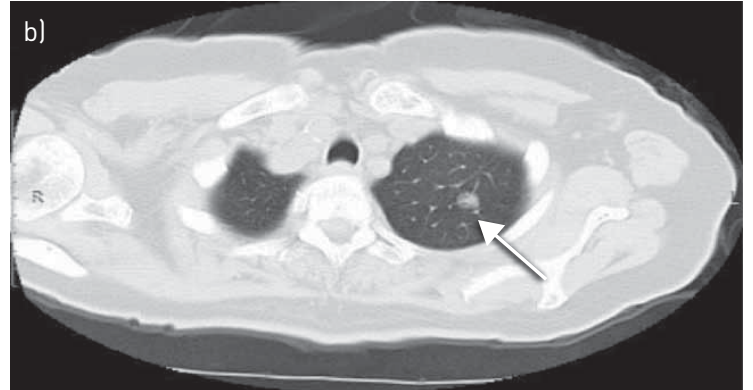

Part-solid nodule

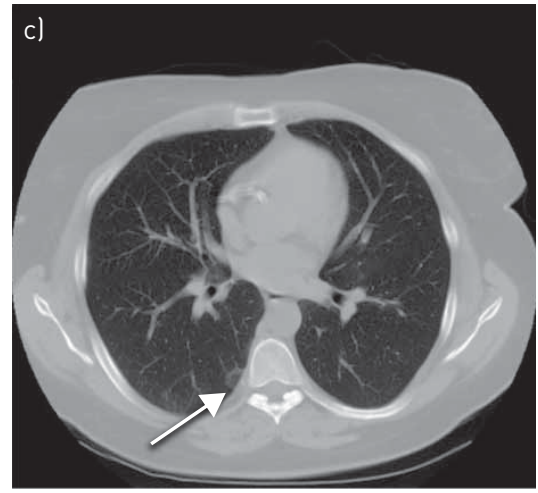

Ground-glass nodule

FIGURE 1 Computed tomography images of different types of solitary pulmonary nodules. 
overall diagnostic yield of $>90 \%$ [14]. CT-TNB is now extensively used in developed countries [15]. However, availability is limited in low-resource settings due to lack of access to CT equipment and few trained operators [16].

Advanced bronchoscopic platforms, including radial endobronchial ultrasound (rEBUS) and navigational bronchoscopy, have improved the diagnostic yield of bronchoscopy for SPNs [17]. rEBUS is a commonly available bronchoscopic imaging modality [18]. It uses a rotating ultrasound transducer to generate $360^{\circ}$ sonographic images of the surrounding lung parenchyma, which enables identification of lesions based on alterations in echogenicity [19]. The rEBUS probe can be delivered within a guide sheath, which serves as an extended working channel, or can be passed via an ultrathin bronchoscope [20] to access the subsegmental airways [21]. Consequently, rEBUS has shown good diagnostic performance in the evaluation of distally situated lesions [22]. Navigational bronchoscopy is an emerging endoscopic technique, which enables navigation to hard-to-reach lesions using superimposed reconstructed three-dimensional CT images [23, 24]. Navigational bronchoscopy systems, such as superDimension Navigation System (Medtronic, Minneapolis, MN, USA), are available in over 800 centres worldwide [25]. However, a barrier to more widespread adoption is cost, with system costs of $€ 145000$ and higher overall procedure costs (€2170), compared with CT-TNB (€1510) [24] and rEBUS (€1680) [26]. The major advantage of rEBUS and navigational bronchoscopy is the lower risk of procedure-related complications compared with CT-TNB, such as pneumothorax (3-5\% versus 23\%) [20, 27]. Multiple bronchoscopic techniques (used in combination) deliver a higher diagnostic yield than each method alone [28]. A recent meta-analysis concluded that rEBUS combined with navigational bronchoscopy may be preferable in sampling SPNs $>2 \mathrm{~cm}$ [29]. This multimodal bronchoscopic approach delivers an acceptable diagnostic yield $(>80 \%)$ with considerably lower risk of adverse events, compared with CT-TNB. Nevertheless, the negative predictive value of navigational bronchoscopy is only 56\% [30], which may be related to sampling inflammatory changes surrounding malignant tissue [31]. Combined navigational bronchoscopy and rEBUS may improve the negative predictive value for malignancy in peripheral lung lesions [32]. However, in many cases a negative biopsy does not provide the clinician with sufficient confidence to discharge the patient from radiological or interventional follow-up.

Fibre-based approaches are compatible with existing bronchoscopic and transthoracic platforms [33-38], and have the potential to augment the diagnostic pathway in SPNs. Optical fibre-based techniques delivered via the working channel of a bronchoscope or via transthoracic needle, permit high-resolution imaging of the distal lung parenchyma [39]. These novel technologies have the capability to identify target biopsy sites, thereby optimising diagnostic accuracy and avoiding repeated procedures. Optical endomicroscopy (OEM), an autofluorescence-based imaging technique, demonstrates abnormal alveolar structure in SPNs in vivo [33-35]. Alternative optical fingerprinting approaches, including time-resolved fluorescence spectroscopy (TRFS), fluorescence-lifetime imaging microscopy (FLIM) and Raman spectroscopy, have shown promise in detecting cancerous lung tissue $[40,41]$. These state-of-the-art optical techniques have the capability to streamline the current care pathway by minimising years of CT surveillance and expediting surgical intervention when necessary. Whilst these are not yet in routine clinical use, they have the potential to deliver in situ diagnostics, and aid immediate treatment decisions in patients with SPNs.

In this review, we discuss the current imaging modalities for evaluating SPNs, including CT and positron emission tomography-computed tomography (PET-CT), and discuss advances in artificial intelligence approaches and molecular imaging. We review optical fibre-based approaches in lung cancer and explore the role that these emerging technologies may play in the field of SPNs.

\section{Computed tomography}

Multi-detector CT has revolutionised the detection and characterisation of SPNs. The evaluation of CT scan appearances plays a key role in the initial assessment of risk of malignancy in SPNs. In addition, CT is the imaging modality of choice for radiological follow-up of SPNs with low risk of malignancy $[1,13,42]$.

Surveillance with serial CT chest imaging is performed in low-risk SPNs, in order to use an assessment of nodule growth to discriminate between benign and malignant SPNs [1]. SPN size has traditionally been assessed by measuring the maximum transverse cross-sectional diameter. However, this approach is limited by poor reliability [43]. Volumetric analysis uses computational algorithms to segment and calculate SPN volumes (expressed as volume doubling time (VDT)). This alternative tool to assess SPN growth has demonstrated improved sensitivity [44,45] and lower false positive rates [46]. Nodule segmentation performance varies across different software platforms [47, 48]; therefore, measurement standardisation will be essential in facilitating future implementation [1].

Malignant SPNs demonstrate a wide range of VDTs. Therefore, establishing a VDT threshold, which differentiates benign from malignant SPNs is challenging. Sub-solid SPNs, encompassing part-solid and 
ground-glass nodules, demonstrate a more indolent growth pattern and confer a good prognosis [49]. These require a less interventional management approach compared with solid SPNs [13]. The application of CT surveillance in sub-solid SPNs is difficult, as the presence of indistinct margins and longer VDTs has implications on frequency and duration of follow-up. The observed VDTs for malignant sub-solid SPNs range between 400 and 1100 days [50], and they may grow after prolonged periods of stability [51]. Therefore, the BTS and Fleischner Society guidelines recommend longer total CT follow-up periods for sub-solid SPNs [1, 13], carrying mean costs of $\sim € 2720$ per patient [52]. The advantage of this watchful waiting approach is the avoidance of unnecessary invasive procedures in individuals with benign disease. However, CT surveillance can have a considerable psychological impact in patients with SPNs. The detection of an SPN can adversely impact upon the patient's quality of life [53], causing anxiety [12] and distress [54]. Patients can initially fear they have underlying lung cancer and may carry the burden of diagnostic uncertainty over many years of follow-up [55].

The number of incidental SPNs is expected to rise due to the widespread use of thoracic CT across multiple disciplines in clinical practice. For instance, cardiac CT has been recommended as a first-line investigation in patients presenting with new-onset chest pain [56, 57]. Some CT scanners limit the field of view (excluding the lungs) to optimise coronary artery spatial resolution. However, a number of centres include the full field of view reconstruction of the thorax, which in turn identifies incidental SPNs [58, 59]. The application of advanced computational methods in radiology are likely to play a key role in the assessment of SPNs. Radiomics, the high-throughput extraction of quantitative features from radiographic images, has shown promise in characterising lung tumour phenotypes, including predicting prognosis [60, 61] and response to therapies [62]. This approach may have a role in predicting malignancy in SPNs [63, 64]. There has also been interest in developing CT image analytics, using artificial intelligence approaches, to enable high-throughput identification and assessment of SPNs [65, 66].

Machine learning is a form of artificial intelligence in which computational algorithms learn to recognise patterns in mass data and make accurate predictions with minimal human intervention. The future implementation of national LDCT screening initiatives will likely result in large numbers of additional SPNs requiring CT surveillance, with consequential impact on workforce and CT scanner capacities [65]. There is great interest in using machine learning techniques to detect and risk stratify SPNs [67, 68]. These automated approaches have the potential to reduce radiologists' workload by avoiding unnecessary follow-up in benign SPNs and enabling earlier identification of malignant SPNs [69]. Researchers at Google designed a deep learning algorithm, which was retrospectively applied to $~ 42000$ lung cancer screening CT scans. They demonstrated that their model performed as well as or better than radiologists in detecting malignant SPNs [70]. BALDWIN et al. [65] applied a machine learning prediction model (using radiological information alone) to assess the risk of malignancy in small SPNs, and demonstrated a sensitivity of $99.5 \%$, outperforming the Brock model risk calculator [9]. Whilst these computer-aided decision supporting technologies hold promise in the field of SPNs, there are number of challenges, including patient selection bias [71], accountability and data privacy issues [72].

\section{Positron emission tomography-computed tomography imaging}

Positron emission tomography (PET) with ${ }^{18} \mathrm{~F}$-fluorodeoxyglucose $\left({ }^{18} \mathrm{~F}-\mathrm{FDG}\right)$, an analogue of glucose, provides functional information based on the increased rates of glucose uptake in cancer cells. Integrated PET-CT scanners offer a synergistic combination of anatomical and metabolic imaging, maintaining the sensitivity of CT and specificity of PET [73]. This imaging modality is commonly used in the diagnosis and staging of lung cancer [74], and now has a key role in the management of SPNs [1]. However, the availability of PET-CT greatly varies throughout the world. For instance there are 6.5 scanners per million people in the USA [75], whereas $>90 \%$ lower-middle income countries do not have access to PET-CT facilities [76]. This is primarily due to high equipment costs (including annual service costs of $\sim € 864000$ ) [77], and limited availability of trained personnel [78].

Current guidelines advocate ${ }^{18}$ F-FDG PET-CT in the further evaluation of high-risk solid SPNs $[1,13]$. HERDER et al. [10] demonstrated that incorporating a qualitative measure of ${ }^{18} \mathrm{~F}$-FDG avidity in an existing validated risk prediction model resulted in improved diagnostic accuracy in SPNs. The addition of ${ }^{18}$ F-FDG PET information increased the area under the curve by $13 \%$ (0.79 to 0.92$)$ [10]. However, this imaging modality has limitations. The pre-test probability of malignancy influences interpretation of PET-CT, with high-risk individuals at risk of false-negative results [42]. Furthermore, the use of ${ }^{18}$ F-FDG PET-CT in subcentimetre SPNs has been unclear, due to limitations of PET-CT spatial resolution [1]. Advances in image reconstruction technologies may enable more accurate PET-CT characterisation of subcentimetre SPNs, in which assessment of metabolic activity is challenging [79].

Image reconstruction methodologies have an impact on the measurement of standardised uptake value (SUV; a relative measure of FDG uptake) in SPNs [80]. The most widely used algorithm, ordered subset 
expectation maximisation (OSEM), generates a reconstructed PET-CT image from the raw data through successive approximations. However, image noise increases with each iteration, which often results in an underestimation of SUV [81]. Advances in PET-CT image reconstruction algorithms, such as Q.Clear (GE Healthcare, Chicago, IL, USA), demonstrate enhanced image quality and improved quantification accuracy, particularly in small SPNs [82]. Furthermore, the implementation of Q.Clear in conjunction with artificial intelligence techniques improves the detection of SPNs in PET-CT, compared with conventional OSEM [83]. Q.Clear yields significantly elevated maximum SUV values in SPNs [82], although this does not alter the diagnostic performance of the Herder model [84].

${ }^{18} \mathrm{~F}-\mathrm{FDG}$ is the most commonly used radiotracer in PET-CT imaging. However, it is not cancer specific, and false-positive uptake is seen in inflammatory or infective conditions [85], which contributes to benign resection rates $(12-15 \%)[86,87]$. Consequently, there have been attempts at developing other PET-CT molecular imaging approaches for SPNs. SCAFOGLIO et al. [88] demonstrated that the activity of the sodium-glucose transporter 2, a potential marker of metabolically active early-stage lung adenocarcinoma, could be imaged in vivo using methyl 4-deoxy-4- $\left[{ }^{18} \mathrm{~F}\right]$ fluoro-alpha-D-glucopyranoside (Me4FDG) PET-CT. Non-approved radiotracers, such as ${ }^{18} \mathrm{~F}$-fluorodeoxythymidine (a tissue proliferation tracer) and ${ }^{11} \mathrm{C}$-methionine (a protein metabolism tracer), have also been investigated [89]. However, the potential of these alternative radiotracers in SPNs remains unclear. For example, a meta-analysis demonstrated ${ }^{18}$ F-fluorodeoxythymidine PET was less sensitive than ${ }^{18} \mathrm{~F}-\mathrm{FDG}$ PET in differentiating benign and malignant lesions [90]. Furthermore, the short half-life of ${ }^{11} \mathrm{C}(20 \mathrm{~min})$, restricts the use of ${ }^{11} \mathrm{C}$-methionine PET to centres with an on-site cyclotron and radiochemistry facilities.

\section{Optical endomicroscopy}

Advanced bronchoscopic techniques, such as rEBUS and navigational bronchoscopy [91, 92], and robotic platforms [93] enable access to most of the lung parenchyma via endobronchial means. This can be further supplemented by transthoracic approaches [94]. However, the diagnostic yield of transbronchial and CT-guided biopsies for small SPNs is influenced by nodule size [29]. Optical endomicroscopy (OEM) enables visualisation of the distal lung parenchyma at high-resolution. Therefore, this novel approach may have a role in augmenting the diagnostic accuracy of existing sampling techniques, such as rEBUS, in the field of SPNs $[33,34]$.

There has been great interest in translating confocal microscopy techniques to enable endomicroscopic exploration of the respiratory system [95]. Fibre-based confocal fluorescence microscopy is an OEM technique, in which the microscope objective luminates a flexible fibre-optic, containing thousands of light-guiding cores, using a laser-scanning unit to scan across the fibre bundle. Each core in the fibre bundle acts as a single pixel in the resulting image with emitted fluorescence travelling back up the same core to a detector [96]. The most commonly used system for pulmonary imaging has a depth of focus of 0-50 $\mu \mathrm{m}$, circular $600 \mu \mathrm{m}$ field of view and scan rate of 12 frames.second $^{-1}$ (Alveoflex, Cellvizio; Mauna Kea Technologies, Paris, France) [97]. The flexibility of the fibre, coupled with the imaging speed, enables real-time microscopic imaging of the distal lung.

OEM can be easily performed during bronchoscopy, with the fibre introduced via the working channel. This provides the clinician with real-time endomicroscopic imaging of the respiratory tract, thereby extending the field of interventional pulmonology to the distal lung and the cellular level [39]. This minimally invasive technique, which adds approximately $10 \mathrm{~min}$ to a conventional bronchoscopy procedure, is well tolerated in topically anaesthetised spontaneously breathing patients [39, 97]. It has a good safety profile and has been studied in numerous respiratory diseases in multiple centres worldwide $[34,35,98,99]$.

At $488 \mathrm{~nm}$ excitation, OEM generates microscopic images of human alveolar structure through the autofluorescence of elastin [39], which represents 50\% of peripheral lung connective tissue fibres [100], and is unaffected by collagen fluorescence [101]. In health, the elastin fibre framework appears as a network of linear contours encircling alveolar ducts and surrounding extra-alveolar micro-vessels. However, this structure can be distorted in distal lung pathologies [102], including SPNs [35] (figure 2).

Label-free OEM pulmonary imaging is an attractive modality for distinguishing normal from abnormal lung tissue. Visual analysis of OEM images in pathological cases demonstrates distortion of the alveolar network, characterised by a tangle of elastin fibres. Advanced image analysis techniques may aid clinicians by enabling automated image classification for the diagnosis of distal lung pathologies [103-105]. HÉBERT et al. [106] analysed OEM images from the distal lung in healthy volunteers and patients with interstitial lung disease. They discriminated healthy alveolar structure from pathological in $86.3 \%$ and $95.1 \%$ of non-smokers and current/ex-smokers, respectively. RАкотомамопуY et al. [107] explored the feasibility of lung cancer detection using OEM with machine learning tools, in patients with bronchial squamous cell 

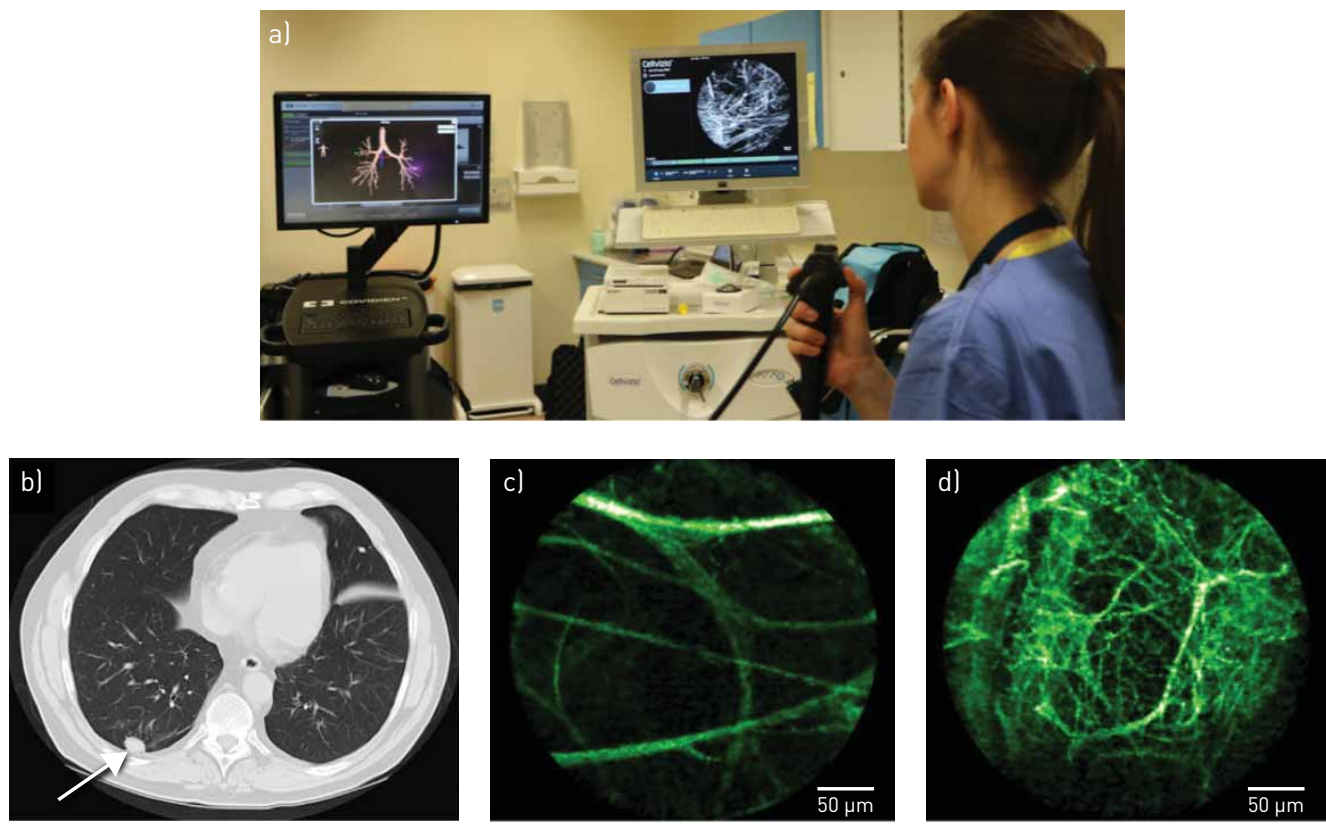

FIGURE 2 a) The delivery of fibre-based optical endomicroscopy (OEM), in conjunction with navigational bronchoscopy, to access a distal solitary pulmonary nodule (SPN) in the clinical setting. Computed tomography (CT) and OEM images from an individual patient presenting with an SPN: b) CT demonstrating solid SPN; c) OEM image demonstrating surrounding healthy elastin structure; d) OEM image demonstrating distorted, abnormal elastin structure within the SPN (subsequently confirmed as benign on histopathological analysis) [35].

carcinoma and healthy volunteers. The authors demonstrated that the application of topical methylene blue (a contrast agent) in conjunction with a $660 \mathrm{~nm}$ OEM system had $90 \%$ classification accuracy in the diagnosis of lung cancer. Whilst these are encouraging studies, they all used very limited datasets. Thus, with larger data accrual, automated computational analysis of OEM images may have a role in the further evaluation of SPNs [108].

Fibre-based OEM, in conjunction with rEBUS and/or navigational bronchoscopy, can access the vast majority of SPNs, including subcentimetre, peripherally located lesions [33-35]. This enables high-resolution imaging of SPNs, including the nodule penumbra which is a key area to sample as intratumoral necrosis is often present [109]. WiJMAns et al. [37] demonstrated that fibre-based OEM, delivered via transthoracic approach, enabled real-time visualisation of pleural abnormalities in mesothelioma, which could be distinguished from benign pleural disease. Fibre-based OEM has the potential to aid identification of optimal biopsy sites, thereby increasing the diagnostic yield and avoiding repeated conventional sampling procedures with associated complications [33-38].

Label-free fibre-based OEM shows benign and malignant SPNs are indistinguishable in vivo, as they both demonstrate abnormal alveolar fluorescence images [35, 110]. SETH et al. [35] evaluated the efficacy of incorporating additional information from OEM in the assessment of risk of malignancy in SPNs (including 66 benign and 25 malignant SPNs). They demonstrated that there were no features, obtained through either manual assessment or automated feature extraction, that significantly improved the operator characteristics of existing SPN risk calculators. The use of contrast agents or fluorescent probes in OEM may play a key role in enabling detailed assessment of risk of malignancy in SPNs.

Exogenous fluorescent contrast agents, such as intravenous fluorescein and topical acriflavine, have been used to enhance bronchial vascular and cellular structure imaging in pulmonary OEM [111-113]. Fluorescein-aided fibre-based OEM enables in vivo imaging of inflammatory and cancer cells in the distal lung parenchyma [114], by enhancing fluorescence of the stromal background [115]. WIJMANs et al. [36] demonstrated the use of an endosonography-guided needle-based OEM system, in conjunction with systemically administered fluorescein, to assess lung cancers and mediastinal lymph nodes. They showed that this needle-based approach enables real-time in vivo visualisation of malignant cells in $~ 90 \%$ of patients with lung cancer (including stage I-IV non-small cell and limited disease small cell lung cancers) [36]. Whilst fluorescein is a safe and widely available contrast agent, one limitation is that it does not stain central airway bronchial epithelial cells [114]. Acriflavine is an alternative fluorescent dye, which strongly labels cell nuclei and superficial epithelial cells. The use of topical acriflavine, in conjunction with 
fibre-based OEM, has demonstrated very high sensitivity and specificity in detecting malignant endobronchial lesions (including non-small cell and small cell lung cancers, and non-neoplastic lesions, such as sarcoidosis) [113]. However, there has been concern regarding the potential carcinogenic effects of acriflavine [116], which has limited further uptake of this technique. Methylene blue is a non-toxic exogenous fluorophore, which enables direct visualisation of cell nuclei, and can be safely used topically to image pre-cancerous lesions in vivo [116]. Methylene blue-aided OEM permits cellular imaging of bronchial cancer [107] and distally situated SPNs [33]. However, in order to give a fluorescence signal, methylene-blue requires a $660 \mathrm{~nm}$ excitation wavelength OEM system. Finally, there has been interest in developing Smartprobes, bespoke compounds which emit fluorescence following activation by specific signalling molecules and proteins, to detect lung pathologies [117-119]. The potential of fluorescein-based Smartprobe imaging in lung cancer has been demonstrated by targeting epidermal growth factor receptor mutations in cell line xenograft mouse model [120] and in ex vivo human lung cancer tissue for visualising matrix activity [118]. Targeted OEM imaging with $488 \mathrm{~nm}$ compatible, topically administered Smartprobes has now commenced in clinical cohorts with confirmed or suspected lung cancer (ClinicalTrials.gov, NCT02676050). The integration of alternative approaches $[118,121]$ and multiplexed molecular imaging [122] makes Smartprobe OEM a promising technique for evaluating malignancy in SPNs.

OEM is an attractive translational technology, which has the potential to augment the diagnostic pathway in SPNs. In the context of pulmonary disease, the FIVE2 device (OptiScan Imaging Ltd, Melbourne, Australia) has been developed for pre-clinical research. This rigid, hand-held instrument, which requires delivery via the transthoracic approach, has been used to evaluate malignant pleural disease in vivo [123]. There is a commercially available flexible fibre-based OEM system (Cellvizio; Mauna Kea Technologies, Paris, France), which is used as a standard diagnostic modality in gastroenterology [124]. It has also received US Food and Drug Administration approval for use with existing endobronchial platforms and transthoracic biopsy needles [125]. The initial OEM system costs are $€ 88000$ [126], and each fibre costs $\sim € 6000$; however, these can be reprocessed for use in up to 20 examinations in multiple patients [107]. This technology has a growing installed user-base ( $>600$ systems worldwide) [127], especially following the introduction of reimbursements for use in gastro-intestinal procedures [124]. The development of single-use OEM fibres [128], and low cost modular imaging systems [122] may herald the wider adoption of fibre-based techniques in the field of interventional pulmonology. Whilst OEM alone aids accurate localisation of SPNs, the addition of novel spectroscopic techniques has the potential to enable identification of malignant features in SPNs.

\section{Time-resolved fluorescence spectroscopy and fluorescence-lifetime imaging microscopy}

Advanced spectroscopic technologies, such as time-resolved fluorescence spectroscopy (TRFS) and fluorescence-lifetime imaging microscopy (FLIM), provide quantifiable biochemical information, which may aid the identification of malignant tissue [129]. These functional methodologies can give a direct insight into the molecular interactions of a fluorophore within its biological environment [130]. Fluorescence intensity, the primary output of OEM, is particularly challenging to quantitively measure in the clinical setting, due to tissue movement [131]. In contrast, fluorescence lifetime, defined as the time a fluorophore spends in excited state before returning to ground state by emitting a photon, is largely independent of external factors [132].

Time-resolved fluorescence spectroscopy techniques detect emitted photons following sample excitation by a pulsed laser source, thereby allowing a lifetime to be calculated for each pixel in a field of view. FLIM delivers highly spatially resolved images (from the contrast in fluorescence lifetimes) to enable the rapid localisation of detected abnormal tissue [133]. To date, time-resolved fluorescence techniques have been relatively slow, limiting clinical implementation. However, this has been overcome by advances in sensor technology [134-136], which break the usual compromise between time-resolution and processing rates, enabling high sensitivity optical fingerprinting. Therefore, there is great interest in clinically translating time-resolved fluorescence techniques for cancer detection $[137,138]$.

Altered metabolism is a hallmark feature of cancer cells. This is characterised by the preferential switch from oxidative phosphorylation to aerobic glycolysis (known as the Warburg effect) [139], which favours tumour growth and proliferation [140]. Metabolic co-factors, such as NADH and FAD, are intrinsically fluorescent and spectrally distinct [132]. Therefore, TRFS and FLIM may enable label-free detection of metabolic changes associated with cancer. SKALA et al. [141] used two-photon FLIM to study NADH and FAD in an in vivo model of epithelial pre-cancer. They demonstrated that the fluorescence lifetime of $\mathrm{NADH}$ decreases in cancerous tissue. More recently, fluorescence lifetimes of NADH and FAD in human non-small cell lung carcinoma cells have been shown to be significantly shorter than in normal cells using 
FLIM [142]. The biochemical basis for these variations is unclear. However, Blacker et al. [143] demonstrated that changes between oxidative and glycolytic metabolism in vitro did not affect $\mathrm{NAD}(\mathrm{P}) \mathrm{H}$ fluorescence decay rates. The lifetime changes observed in cancers may reflect shifts in NADPH/NADH balance.

Label-free fluorescence spectroscopy and imaging have been recognised as potential tools for the detection of pre-cancer/cancer in ex vivo human tissue with high specificity [144]. FLIM endoscopy has been used to identify oral pre-cancerous and cancerous lesions, (such as epithelial dysplasia and squamous cell carcinoma) from benign inflammatory conditions in vivo [145]. Furthermore, WANG et al. [40] investigated the ability of FLIM to differentiate lung cancer from healthy tissue $(5 \mathrm{~cm}$ from tumour margin) in ex vivo human lung specimens. They demonstrated that the fluorescence lifetime of cancerous lung tissues is consistently lower than normal tissues, which they apportioned to the decrease in both NADH and FAD lifetimes. They reported excellent sensitivity and specificity for the detection of lung cancer $(92.9 \%$ and 92.3\% respectively) using FLIM. Therefore, this novel spectroscopic technique may have a role in the detection of lung cancer, in particular the evaluation of risk of malignancy in SPNs.

Commercially available FLIM devices, such as DermaInspect and MPTflex Multiphoton Laser Tomography ( JenLab GmbH, Berlin, Germany), have been used to study superficial tumours in vivo, such as malignant melanoma [146] and glioblastoma (during craniotomy) [147]. However, these microscope systems are not deliverable to deeper sited tissues, such as the lungs. Kennedy et al. [148] have demonstrated confocal FLIM endomicroscopy by incorporating FLIM with Cellvizio fibre-based OEM system, which enabled label-free, real-time imaging of live cells using $488 \mathrm{~nm}$ excitation. Thus, fibre-based dual fingerprinting techniques have the potential to provide new diagnostic imaging modalities for SPNs, with the aim of enabling real-time verification of malignant SPNs (supplementary video).
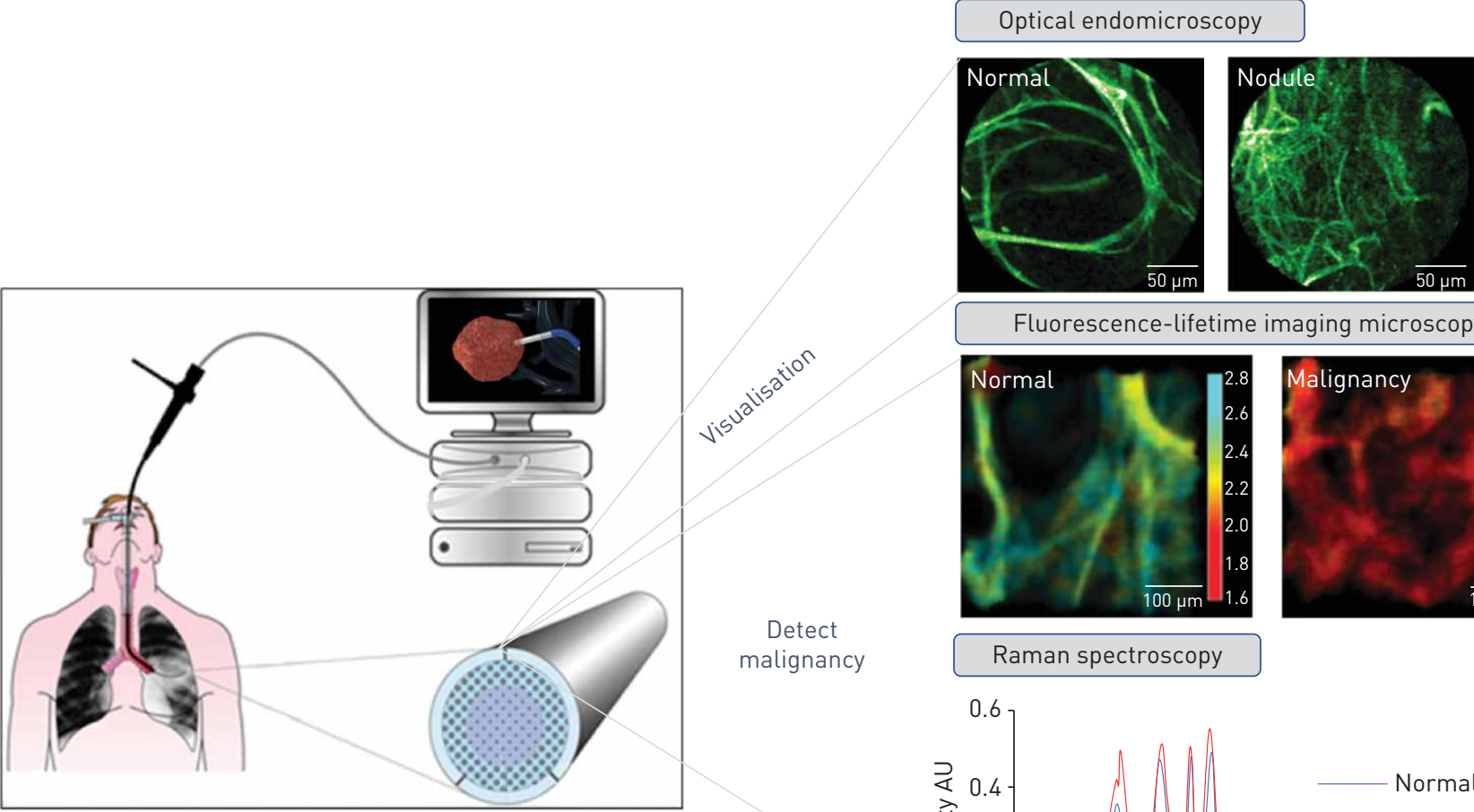

Fluorescence-lifetime imaging microscopy
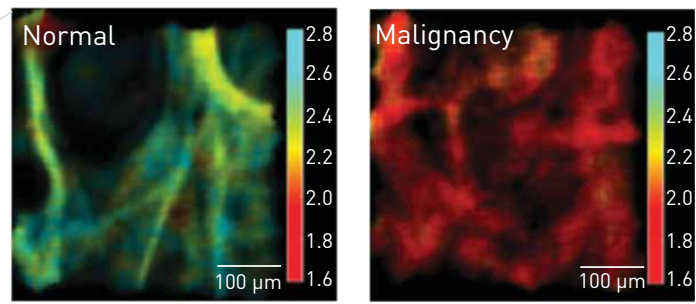

Detect malignancy

Raman spectroscopy

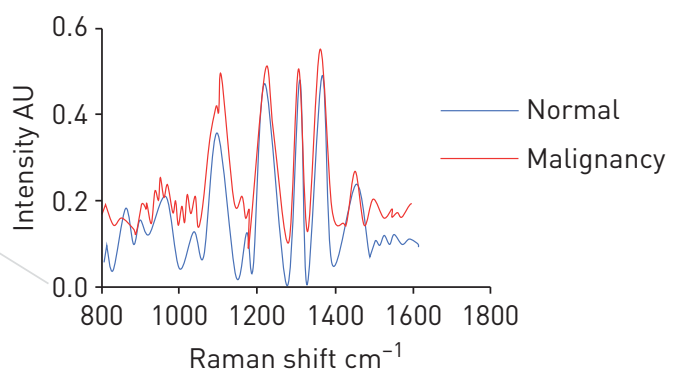

FIGURE 3 Overview of optical fibre-based technologies, delivered via the working channel of a bronchoscope to access and characterise a solitary pulmonary nodule. 


\section{Raman Spectroscopy}

Raman spectroscopy is a label-free optical technique, based on the inelastic scattering of light. Raman spectra contain information on the vibrational and rotational energy transitions of molecules [149], thereby yielding a unique optical fingerprint of the molecular composition of a biological sample, including ex vivo lung cancer [150].

Raman spectroscopy is a promising technique for detecting lung cancer in vivo [151] due to its ability to detect biochemical changes associated with malignancy, such as higher metabolic activity and changes in lipid and protein levels [152]. Identifying changes in Raman spectra using visual observation can be challenging. However, the recent introduction of machine learning approaches in Raman spectroscopy has demonstrated excellent accuracy in differentiating lung cancer, from healthy lung tissue ex vivo [153]. To date, the clinical translation of Raman technology has been limited. Raman scattering is an inherently weak process as only a very small proportion of photons are inelastically scattered. Thus, high intensity illumination and long acquisition times are usually required [154]. Recently, McGregor et al. [41] demonstrated the application of a real-time endoscopic Raman spectroscopy system in evaluating normal, inflamed, dysplastic and malignant bronchial tissue (confirmed histologically) in vivo. They reported excellent sensitivity (90\%) in detecting early lung malignancies. The Raman signatures of inflamed bronchial tissue were also considerably different, compared with other pathologies, thereby highlighting the potential role of Raman spectroscopy in the characterisation of SPNs, the vast majority of which are benign.

The development of a miniature Raman probe, delivered via the working channel of a bronchoscope, has enabled acquisition of in vivo Raman spectra from the peripheral lung, including normal tissue and SPNs [155]. Furthermore, the development of fibre-based multimodal platforms is underway [156, 157], with the aim of enabling real-time diagnostic imaging and spectroscopic identification of malignant SPNs in situ (figure 3).

\section{Conclusion}

SPNs are a clinical and diagnostic challenge. Whilst the majority are benign, some represent early treatable lung cancer. Existing care pathways involve endobronchial/transthoracic tissue biopsies or radiological surveillance, which can be associated with suboptimal diagnostic yield, procedure-related complications and patient anxiety. Recent advances in artificial intelligence approaches in CT and PET-CT hold promise in enabling accurate characterisation of SPNs. Fibre-based optical fingerprinting approaches, in conjunction with endobronchial and transthoracic platforms, may facilitate advanced diagnostic capabilities in patients presenting with SPNs. Autofluorescence OEM has demonstrated aberrant alveolar fluorescence structure in SPNs in vivo. Furthermore, novel spectroscopic approaches, such as TRFS, FLIM and Raman spectroscopy, have shown promise in distinguishing malignancy from normal human lung tissue. Fibre-based technologies have the potential to enable in situ characterisation and real-time microscopic imaging of SPNs, thereby streamlining the diagnostic pathway by identifying individuals with malignant SPNs early and expediting curative treatment.

Conflict of interest: S. Fernandes reports grants from MRC and Boston Scientific, during the conduct of the study. G. Williams has patents planned relating to FLIM. E. Williams has patents planned relating to FLIM. K. Ehrlich has nothing to disclose. J. Stone reports grants from EPSRC and Boston Scientific, during the conduct of the study; and has a patent imaging fibre issued. N. Finlayson reports grants from EPSRC, during the conduct of the study; other from Prothea-X, outside the submitted work. M. Bradley reports grants from MRC, Boston Scientific and EPSRC, during the conduct of the study. R.R. Thomson reports grants from University of Edinburgh, during the conduct of the study. A.R. Akram reports academic grants, during the conduct of the study. K. Dhaliwal reports grants from MRC, Boston Scientific and EPSRC, and reimbursment for travel and consultancy from Mauna Kea Technologies, during the conduct of the study.

Support statement: This work was supported by Cancer Research UK (grant: A24867), the Engineering and Physical Sciences Research Council (grants: EP/K03197X/1 and EP/S001123/1) and the Medical Research Council (grant: MR/ R017794/1). Funding information for this article has been deposited with the Crossref Funder Registry.

\section{References}

1 Callister MEJ, Baldwin DR, Akram AR, et al. BTS Guidelines for the Investigation and Management of Pulmonary Nodules. Thorax 2015; 70: iil-iis4.

2 Field JK, Duffy SW, Baldwin DR, et al. UK Lung Cancer RCT Pilot Screening Trial: baseline findings from the screening arm provide evidence for the potential implementation of lung cancer screening. Thorax 2016; 71: 161-170.

3 Ferlay J, Soerjomataram I, Dikshit R, et al. Cancer incidence and mortality worldwide: sources, methods and major patterns in GLOBOCAN 2012. Int J Cancer 2015; 136: E359-E386.

4 Detterbeck FC, Boffa DJ, Kim AW, et al. The Eighth Edition Lung Cancer Stage Classification. Chest 2017; 151: 193-203. 

low-dose computed tomographic screening. N Engl J Med 2011; 365: 395-409. de Koning HJ, Van Der Aalst CM, De Jong PA, et al. Reduced lung-cancer mortality with volume CT screening in a randomized trial. $N$ Engl J Med 2020; 382: 503-513.

7 NHS England - National Cancer Programme. Targeted Screening for Lung Cancer with Low Radiation Dose Computed Tomography. London, NHS England, 2019; pp. 1-30.

8 Oudkerk M, Devaraj A, Vliegenthart R, et al. European position statement on lung cancer screening. Lancet Oncol 2017; 18: e754-e766.

9 McWilliams A, Tammemagi MC, Mayo JR, et al. Probability of cancer in pulmonary nodules detected on first screening CT. N Engl J Med 2013; 369: 910-919.

10 Herder GJ, van Tinteren $\mathrm{H}$, Golding RP, et al. Clinical prediction model to characterize pulmonary nodules: validation and added value of $18 \mathrm{~F}$-fluorodeoxyglucose positron emission tomography. Chest 2005; 128: 2490-2496.

11 Lokhandwala T, Dann R, Johnson M, et al. Costs of the diagnostic workup for lung cancer: a Medicare claims analysis. IJROBP 2014; 90: S9-S10.

12 Byrne MM, Weissfeld J, Roberts MS. Anxiety, fear of cancer, and perceived risk of cancer following lung cancer screening. Med Decis Mak 2008; 28: 917-925.

13 MacMahon H, Naidich DP, Goo JM, et al. Guidelines for management of incidental pulmonary nodules detected on CT images: from the Fleischner Society 2017. Radiology 2017; 284: 228-243.

14 Zhang H, Zeng X, Xing F, et al. The diagnostic accuracy of CT-guided percutaneous core needle biopsy and fine needle aspiration in pulmonary lesions: a meta-analysis. Clin Radiol 2016; 71: e1-e10.

15 The Royal College of Radiologists. Percutaneous lung biopsy - Safety and Diagnostic Adequacy. London, The Royal College of Radiologists, 2009; pp. 1-4.

16 Dobbins J III, Frush D, Kigongo C, et al. Medical imaging safety in global health radiology. In: Mollura DJ, Culp MP, Lungren MP, eds. Radiology in Global Health. 2nd Edn. Switzerland, Springer, 2019; pp. 85-106.

17 Wang Memoli J, Nietert P, Silvestri G. Meta-analysis of guided bronchoscopy for the evaluation of the pulmonary nodule. Chest 2012; 142: 385-393.

18 Bennett H, Verma R. British Thoracic Society National Audit Report: Adult Bronchoscopy Audit 2017. BTS Rep 2018; 9: 1-10.

19 Dhillon S, Harris K. Bronchoscopy for the diagnosis of peripheral lung lesions. J Thorac Dis 2017; 9 S1047-S1058.

20 Oki M, Saka H, Kitagawa C, et al. Randomized study of endobronchial ultrasound-guided transbronchial biopsy: thin bronchoscopic method versus guide sheath method. J Thorac Oncol 2012; 7: 535-541.

21 Oki M, Saka H, Asano F, et al. Use of an ultrathin vs thin bronchoscope for peripheral pulmonary lesions: a randomized trial. Chest 2019; 156: 954-964.

22 Steinfort D, Khor Y, Manser R, et al. Radial probe endobronchial ultrasound for the diagnosis of peripheral lung cancer: systematic review and meta-analysis. Eur Respir J 2011; 37: 902-910.

23 Rivera MP, Mehta AC, Wahidi MM. Establishing the diagnosis of lung cancer: diagnosis and management of lung cancer, 3rd ed: American College of Chest Physicians evidence-based clinical practice guidelines. Chest 2013; 143: e142S-e165S.

24 National Institute for Health and Care Excellence (NICE). superDimension Navigation System to help diagnostic sampling of peripheral lung lesions: Medtech innovation briefing [MIB194]. London, NICE, 2019; pp. 1-17.

25 Medtronic. Superdimension navigation system with fluoroscopic navigation technology. Date last updated: Aug 20, 2020. Date last accessed: Aug 20, 2020. www.medtronic.com/content/dam/covidien/library/us/en/product/ interventional-lung-solutions/superdimension-navigation-system-overview-brochure.pdf

26 Steinfort D, Liew D, Irving L. Radial probe EBUS versus CT-guided needle biopsy for evaluation of peripheral pulmonary lesions: an economic analysis. Eur Respir J 2013; 41: 539-547.

27 Deng CJ, Dai FQ, Qian K, et al. Clinical updates of approaches for biopsy of pulmonary lesions based on systematic review. BMC Pulm Med 2018; 18: 146.

28 Ishida T, Asano F, Yamazaki K, et al. Virtual Navigation in Japan Trial Group. Virtual bronchoscopic navigation combined with endobronchial ultrasound to diagnose small peripheral pulmonary lesions: a randomised trial. Thorax 2011; 66: 1072-1077.

29 Han Y, Kim HJ, Kong KA, et al. Diagnosis of small pulmonary lesions by transbronchial lung biopsy with radial endobronchial ultrasound and virtual bronchoscopic navigation versus CT-guided transthoracic needle biopsy: a systematic review and meta-analysis. PLoS One 2018; 13: e0191590.

30 Folch E, Prichett M, Nead M, et al. Electromagnetic navigation bronchoscopy for peripheral pulmonary lesions: One-year results of the prospective, multicenter NAVIGATE study. J Thorac Oncol 2019; 14: 445-458.

31 Gex G, Pralong J, Combescure C, et al. Diagnostic yield and safety of electromagnetic navigation bronchoscopy for lung nodules: a systematic review and meta-analysis. Respiration 2014; 87: 165-176.

32 Eberhardt R, Anantham D, Ernst A, et al. Multimodality bronchoscopic diagnosis of peripheral lung lesions: a randomized controlled trial. Am J Respir Crit Care Med 2007; 176: 36-41.

33 Hassan T, Piton N, Lachkar S, et al. A novel method for in vivo imaging of solitary lung nodules using navigational bronchoscopy and confocal laser microendoscopy. Lung 2015; 193: 773-778.

34 Hassan T, Thiberville L, Hermant C, et al. Assessing the feasibility of confocal laser endomicroscopy in solitary pulmonary nodules for different part of the lungs, using either 0.6 or $1.4 \mathrm{~mm}$ probes. PLoS One 2017; 12: e0189846.

35 Seth S, Akram AR, McCool P, et al. Assessing the utility of autofluorescence-based pulmonary optical endomicroscopy to predict the malignant potential of solitary pulmonary nodules in humans. Sci Rep 2016; 6: 31372 .

36 Wijmans L, Yared J, de Bruin DM, et al. Needle-based confocal laser endomicroscopy for real-time diagnosing and staging of lung cancer. Eur Respir J 2019; 53: 1801520.

37 Wijmans L, Baas P, Sieburgh TE, et al. Confocal laser endomicroscopy as a guidance tool for pleural biopsies in malignant pleural mesothelioma. Chest 2019; 156: 754-763. 
Shulimzon TR, Lieberman S. Feasibility of confocal laser microscopy in CT-guided needle biopsy of pulmonary and mediastinal tumors: a proof-of-concept pilot study. J Vasc Interv Radiol 2016; 27: 275-280.

Thiberville L, Salaün M, Lachkar S, et al. Human in vivo fluorescence microimaging of the alveolar ducts and sacs during bronchoscopy. Eur Respir J 2009; 33: 974-985.

Wang M, Tang F, Pan X, et al. Rapid diagnosis and intraoperative margin assessment of human lung cancer with fluorescence lifetime imaging microscopy. BBA Clin 2017; 8: 7-13.

McGregor HC, Short MA, McWilliams A, et al. Real-time endoscopic Raman spectroscopy for in vivo early lung cancer detection. J Biophotonics 2017; 10: 98-110.

Gould MK, Donington J, Lynch WR, et al. Evaluation of individuals with pulmonary nodules: when is it lung cancer? Diagnosis and management of lung cancer, 3rd ed: American College of Chest Physicians evidence-based clinical practice guidelines. Chest 2013; 143: e93S-e120S.

Revel M-P, Bissery A, Bienvenu M, et al. Are two-dimensional CT measurements of small noncalcified pulmonary nodules reliable? Radiology 2004; 231: 453-458.

Revel MP, Merlin A, Peyrard S, et al. Software volumetric evaluation of doubling times for differentiating benign versus malignant pulmonary nodules. Am J Roentgenol 2006; 187: 135-142.

Ko JP, Berman EJ, Kaur M, et al. Pulmonary nodules: Growth rate assessment in patients by using serial CT and three-dimensional volumetry. Radiology 2012; 262: 662-671.

Horeweg N, van der Aalst CM, Vilegenthart R, et al. Volumetric computed tomography screening for lung cancer: three rounds of the NELSON trial. Eur Respir J 2013; 42: 1659-1667.

de Hoop B, Gietema H, van Ginneken B, et al. A comparison of six software packages for evaluation of solid lung nodules using semi-automated volumetry: what is the minimum increase in size to detect growth in repeated CT examinations. Eur Radiol 2009; 19: 800-808.

Zhao YR, van Ooijen P, Dorrius M, et al. Comparison of three software systems for semi-automatic volumetry of pulmonary nodules on baseline and follow-up CT examinations. Acta Radiol 2014; 55: 691-698.

Scholten ET, de Jong PA, de Hoop B, et al. Towards a close computed tomography monitoring approach for screen detected subsolid pulmonary nodules? Eur Respir J 2015; 45: 765-773.

Li J, Xia T, Yang X, et al. Malignant solitary pulmonary nodules: assessment of mass growth rate and doubling time at follow-up CT. J Thorac Dis 2018; 10: S797-S806.

Lindell RM, Hartman TE, Swensen SJ, et al. 5-year lung cancer screening experience: growth curves of 18 lung cancers compared to histologic type, CT attenuation, stage, survival, and size. Chest 2009; 136: 1586-1595.

Hammer M, Palazzo L, Paquette A, et al. Cost-effectiveness of follow-up for subsolid pulmonary nodules in high-risk patients. J Thorac Oncol 2020; 15: 1298-1305.

Lemonnier I, Baumann C, Jolly D, et al. Solitary pulmonary nodules: consequences for patient quality of life. Qual Life Res 2011; 20: 101-109.

Slatore C, Soylemez Wiener R, Golden S, et al. Longitudinal assessment of distress among veterans with incidental pulmonary nodules. Ann Am Thorac Soc 2016; 13: 1983-1991. an indeterminate pulmonary nodule. Heal Expect 2015; 18: 355-365. coronary heart disease (SCOT-HEART): an open-label, parallel-group, multicentre trial. Lancet 2015; 385: 2383-2391.

Moss AJ, Williams MC, Newby DE, et al. The Updated NICE Guidelines: cardiac CT as the first-line test for coronary artery disease. Curr Cardiovasc Imaging Rep 2017; 10: 15.

Koonce J, Schoepf JU, Nguyen SA, et al. Extra-cardiac findings at cardiac CT: experience with 1,764 patients. Eur Radiol 2009; 19: 570-576.

Robertson J, Nicholls S, Bardin P, et al. Incidental pulmonary nodules are common on CT coronary angiogram and have a significant cost impact. Hear Lung Circ 2019; 28: 295-301.

Huang Y, Liu Z, He L, et al. Radiomics signature: a potential biomarker for the prediction of disease-free survival in early-stage (I or II) non-small cell lung cancer. Radiology 2016; 281: 947-957. quantitative radiomics approach. Nat Commun 2014; 5: 4006

Aerts H, Grossmann P, Tan Y, et al. Defining a radiomic response phenotype: a pilot study using targeted therapy in NSCLC. Sci Rep 2016; 6: 33860.

Chen $\mathrm{CH}$, Chang $\mathrm{CK}$, Tu CY, et al. Radiomic features analysis in computed tomography images of lung nodule classification. PLoS One 2018; 13: e0192002.

Choi W, Oh J, Riyahi S, et al. Radiomics analysis of pulmonary nodules in low-dose CT for early detection of lung cancer. Med Phys 2018; 45: 1537-1549.

Baldwin DR, Gustafson J, Pickup L, et al. External validation of a convolutional neural network artificial intelligence tool to predict malignancy in pulmonary nodules. Thorax 2020; 75: 306-312.

Ather S, Kadir T, Gleeson F. Artificial intelligence and radiomics in pulmonary nodule management: current status and future applications. Clin Radiol 2020; 75: 13-19.

Yang Y, Feng X, Chi W, et al. Deep learning aided decision support for pulmonary nodules diagnosing: a review. J Thorac Dis 2018; 10: S867-S875.

Huang P, Lin CT, Li Y, et al. Prediction of lung cancer risk at follow-up screening with low-dose CT: a training and validation study of a deep learning method. Lancet Digit Heal 2019; 1: E353-E362.

Massion PP, Antic S, Ather S, et al. Assessing the accuracy of a deep learning method to risk stratify indeterminate pulmonary nodules. Am J Respir Crit Care Med 2020; 202: 241-249.

Ardila D, Kiraly AP, Bharadwaj S, et al. End-to-end lung cancer screening with three-dimensional deep learning on low-dose chest computed tomography. Nat Med 2019; 25: 954-961.

Kadir T, Gleeson F. Lung cancer prediction using machine learning and advanced imaging techniques. Transl Lung Cancer Res 2018; 7: 304-312.

Burki TK. The role of AI in diagnosing lung diseases. Lancet Respir Med 2019; 7: 1015-1016.

Kim S, Allen-Auerback M, Goldin J, et al. Accuracy of PET/CT in characterization of solitary pulmonary lesions. J Nucl Med 2007; 48: 214-220. 
National Institute for Health and Care Excellence (NICE). Lung Cancer: Diagnosis and Management. London, NICE, 2019; pp. 1-40.

Buck A, Herrman K, Stargardt T, et al. Economic evaluation of PET and PET/CT in oncology: evidence and methodologic approaches. J Nucl Med Technol 2010; 38: 6-17.

World Health Organization (WHO). Global Atlas of Medical Devices. Geneva, WHO, 2017; pp. 1-480.

Gajuryal SH, Daga A, Siddharth V, et al. Unit cost analysis of PET-CT at an apex public sector health care institute in India. Indian J Nucl Med 2017; 32: 1-6.

Verduzco-Aguirre H, Lopes G, Soto-Perez-De-Celis S. Implementation of diagnostic resources for cancer in developing countries: a focus on PET/CT. Ecancermedicalscience 2019; 13: ed87.

Sim Y, Poon F. Imaging of solitary pulmonary nodule; a clinical review. Quant Imaging Med Surg 2013; 3: 316-326.

Riegler G, Karanikas G, Rausch I, et al. Influence of PET reconstruction technique and matrix size on qualitative and quantitative assessment of lung lesions on [18F]-FDG-PET: a prospective study in 37 cancer patients. Eur J Radiol 2017; 90: 20-26.

Tragardh E, Minarik D, Almquist H, et al. Impact of acquisition time and penalizing factor in a block-sequential regularized expectation maximization reconstruction algorithm on a Si-photomultiplier-based PET-CT system for 18F-FDG. EJNMMI Res 2019; 9: 64.

Teoh EJ, McGowan DR, Bradley KM, et al. Novel penalised likelihood reconstruction of PET in the assessment of histologically verified small pulmonary nodules. Eur Radiol 2016; 26: 576-584.

Schwyzer M, Martini K, Benz DC, et al. Artificial intelligence for detecting small FDG-positive lung nodules in digital PET/CT: impact of image reconstructions on diagnostic performance. Eur Radiol 2020; 30: 2031-2040.

Murphy D, Royle L, Chalampalakis Z, et al. The effect of a novel Bayesian penalised likelihood PET reconstruction algorithm on the assessment of malignancy risk in solitary pulmonary nodules according to the British Thoracic Society guidelines. Eur J Radiol 2019; 117: 149-155.

Feng M, Yang X, Ma Q, et al. Retrospective analysis for the false positive diagnosis of PET-CT scan in lung cancer patients. Medicine (Baltimore) 2017; 96: e7415.

Petersen RH, Hansen HJ, Dirksen A, et al. Lung cancer screening and video-assisted thoracic surgery. J Thorac Oncol 2012; 7: 1026-1031. solitary pulmonary nodules. Ann Thorac Surg 2012; 93: 266-273.

Scafoglio CR, Villegas B, Abdelhady G, et al. Sodium-glucose transporter 2 is a diagnostic and therapeutic target for early-stage lung adenocarcinoma. Sci Transl Med 2018; 10: eaat5933.

Maffione AM, Grassetto G, Rampin L, et al. Molecular imaging of pulmonary nodules. Am J Roentgenol 2014; 202: W217-W223.

Li XF, Dai D, Song XY, et al. Comparison of the diagnostic performance of $18 \mathrm{~F}$-fluorothymidine versus 18F-fluorodeoxyglucose positron emission tomography on pulmonary lesions: a meta analysis. Mol Clin Oncol 2015; 3: 101-108

Chen A, Chenna P, Loiselle A, et al. Radial probe endobronchial ultrasound for peripheral pulmonary lesions: a 5-year institutional experience. Ann Am Thorac Soc 2014; 11: 578-582. bronchoscopy for lung nodules. J Thorac Dis 2015; 7: 799-809.

Chen A, Gillespie C. Robotic Endoscopic Airway Challenge: REACH Assessment. Ann Thorac Surg 2018; 106: 293-297.

Di Bardino DM, Yarmus LB, Semaan RW. Transthoracic needle biopsy of the lung. J Thorac Dis 2015; 7: S304-S316.

Bourg-Heckly G, Thiberville L, Vever-Bizet C, et al. In vivo endoscopic autofluorescence microspectro-imaging of bronchi and alveoli. Proc SPIE 2008: 6851: 685104.

Perperidis A, Dhaliwal K, Mclaughlin S, et al. Image computing for fibre-bundle endomicroscopy: a review. Med Image Anal 2020: 62: 101620.

Thiberville L, Salaün M, Lachkar S, et al. Confocal fluorescence endomicroscopy of the human airways. Proc Am Thorac Soc 2009; 6: 444-449.

Meng P, Tan GL, Low SY, et al. Fibred confocal fluorescence microscopy in the diagnosis of interstitial lung diseases. J Thorac Dis 2016; 8: 3505-3514.

Yick $\mathrm{CY}$, von der Thüsen $\mathrm{JH}$, Bel $\mathrm{EH}$, et al. In vivo imaging of the airway wall in asthma: fibered confocal fluorescence microscopy in relation to histology and lung function. Respir Res 2011; 12: 85. 756-765.

Thiberville L, Moreno-Swirc S, Vercauteren T, et al. In vivo imaging of the bronchial wall microstructure using fibered confocal fluorescence microscopy. Am J Respir Crit Care Med 2007; 175: 22-31. interstitial lung diseases: specific descriptors and correlation with chest CT. Respirology 2019; 24: 783-791.

Désir C, Petitjean C, Heutte L, et al. An SVM-based distal lung image classification using texture descriptors. Comput Med Imaging Graph 2012; 36: 264-270.

Désir C, Petitjean C, Heutte L, et al. Classification of endomicroscopic images of the lung based on random subwindows and extra-trees. IEEE Trans Biomed Eng 2012; 59: 2677-2683.

Bondesson D, Schneider MJ, Silbernagel E, et al. Automated evaluation of probe-based confocal laser endomicroscopy in the lung. PLoS One 2020; 15: e0232847.

Hébert D, Désir C, Petitjean C, et al. Detection of pathological condition in distal lung images. IEEE 2012; 9: 1603-1606.

Rakotomamonjy A, Petitjean C, Salaün M, et al. Scattering features for lung cancer detection in fibered confocal fluorescence microscopy images. Artif Intell Med 2014; 61: 105-118.

Perperidis A, Akram AR, Altmann Y, et al. Automated detection of uninformative frames in pulmonary optical endomicroscopy. IEEE Trans Biomed Eng 2017; 64: 87-98. 

in incremental dynamic computed tomography analysis of solitary pulmonary nodules? J Thorac Imaging 1997; 12: 181-187.

110 Su Z, Zhong C, Li S, et al. Needle-based confocal laser endomicroscopy in the diagnosis of peripheral pulmonary nodule: a preliminary report. J Thorac Dis 2017; 9: 2608-2612.

111 Suter M, McLennan G, Reinhardt J, et al. Bronchoscopic imaging of pulmonary mucosal vasculature responses to inflammatory mediators. J Biomed Opt 2005; 10: 034013.

112 Wijmans L, de Bruin DM, Meijer SL, et al. Real-time optical biopsy of lung cancer. Am J Respir Crit Care Med 2016; 194: e10-e11.

113 Fuchs FS, Zirlik S, Hildner K, et al. Confocal laser endomicroscopy for diagnosing lung cancer in vivo. Eur Respir J 2013; 41: 1401-1408

114 Fuchs FS, Zirlik S, Hildner K, et al. Fluorescein-aided confocal laser endomicroscopy of the lung. Respiration 2011; 81: 32-38.

115 Goorsenberg A, Kalverda K, Annema JT, et al. Advances in optical coherence tomography and confocal laser endomicroscopy in pulmonary diseases. Respiration 2020; 99: 190-205.

116 Obstoy B, Salaün M, Veresezan L, et al. Safety and performance analysis of acriflavine and methylene blue for in vivo imaging of precancerous lesions using fibered confocal fluorescence microscopy (FCFM): an experimental study. BMC Pulm Med 2015; 15: 30.

117 Akram AR, Avlonitis N, Lilienkampf A, et al. A labelled-ubiquicidin antimicrobial peptide for immediate in situ optical detection of live bacteria in human alveolar lung tissue. Chem Sci 2015; 6: 6971-6979.

118 Megia-Fernandez A, Mills B, Michels C, et al. Bimodal fluorogenic sensing of matrix proteolytic signatures in lung cancer. Org Biomol Chem 2018; 16: 8056-8063.

119 Akram AR, Chankeshwara SV, Scholefield E, et al. In situ identification of Gram-negative bacteria in human lungs using a topical fluorescent peptide targeting lipid A. Sci Transl Med 2018; 10: eaal0033.

120 Patout M, Guisier F, Brune X, et al. Real-time molecular optical micro-imaging of EGFR mutations using a fluorescent erlotinib based tracer. BMC Pulm Med 2019; 19: 3.

121 Staderini M, Megia-Fernandez A, Dhaliwal K, et al. Peptides for optical medical imaging and steps towards therapy. Bioorganic Med Chem 2018; 26: 2816-2826.

122 Krstajić N, Mills B, Murray I, et al. Low-cost high sensitivity pulsed endomicroscopy to visualize tricolor optical signatures. J Biomed Opt 2018; 23: 076005.

123 Mitchell P, Knight S, Crowley P, et al. Intra-operative scanning confocal endomicroscopy of pleural disease: in vivo diagnosis of malignancy. $J$ Thorac Oncol 2012; 7: 101-O12.

124 Wang KK, Carr-Locke DL, Singh SK, et al. Use of probe-based confocal laser endomicroscopy (pCLE) in gastrointestinal applications. A consensus report based on clinical evidence. United Eur Gastroenterol J 2015; 3: 230-254.

125 Mauna Kea Technologies. Mauna Kea Technologies Receives 510(k) Clearance for the Cellvizio Needle-based-AQ-Flex 19 Confocal Miniprobe Enabling Peripheral Lung Nodule Targeting and Imaging. Date last updated: Feb 25, 2019. Date last accessed: Aug 20, 2020. www.maunakeatech.com/en/news-events/135mauna-kea-technologies-rec-oit-l-autorisation-de-la-fda-pour-l-utilisation-du-cellvizio-permettant-le-ciblage-et-1imagerie-des-nodules-pulmonaires-pe-riphe-riques

126 National Institute for Health and Care Excellence (NICE). Cellvizio confocal endomicroscopy system for characterising pancreatic cysts: Medtech innovation briefing [MIB69]. London, NICE, 2016; pp. 1-32. Mauna Kea Technologies. Bringing the microscope and pathology lab of the future inside the patient. Date last updated: Oct 22, 2019. Date last accessed: Aug 20, 2020. www.maunakeatech.com/en/about-us/17-our-story

128 Stone JM, Wood HAC, Harrington K, et al. Low index contrast imaging fibers. Opt Lett 2017; 42: 1484-1487.

129 McGinty J, Galletly NP, Dunsby C, et al. Wide-field fluorescence lifetime imaging of cancer. Biomed Opt Express 2010; 1: 627-640.

130 Becker W. Fluorescence lifetime imaging: techniques and applications. J Microsc 2012; 247: $119-136$.

131 Marcu L. Fluorescence lifetime techniques in medical applications. Ann Biomed Eng 2012; 40: 304-331.

132 Berezin MMY, Achilefu S. Fluorescence lifetime measurements and biological imaging. Chem Rev 2010; 110: 2641-2684.

133 Sherman AJ, Papour A, Bhargava S, et al. Normalized fluorescence lifetime imaging for tumor identification and margin delineation. Proc SPIE 2013; 8572: 85721H.

134 Kufcsák A, Erdogan A, Walker R, et al. Time-resolved spectroscopy at 19,000 lines per second using a CMOS SPAD line array enables advanced biophotonics applications. Opt Express 2017; 25: 11103-11123.

135 Erdogan A, Walker R, Finlayson N, et al. A CMOS SPAD line sensor with per-pixel histogramming TDC for time-resolved multispectral imaging. IEEE J Solid-State Circuits 2019; 54: 1705-1719.

136 Henderson R, Johnston N, Mattioli della Rocca F, et al. A 192×128 Time Correlated SPAD Image Sensor in 40-nm CMOS Technology. IEEE J Solid-State Circuits 2019; 54: 1907-1916.

137 Sun Y, Hatami N, Yee M, et al. Fluorescence lifetime imaging microscopy for brain tumor image-guided surgery. J Biomed Opt 2010; 15: 056021.

138 Sun Y, Phipps JE, Meier J, et al. Endoscopic fluorescence lifetime imaging for in vivo intraoperative diagnosis of oral cancer. Microsc Microanal 2013; 19: 791-798.

139 Warburg O. On the origin of cancer cells. Science 1956; 123: 309-314.

140 Jones R, Thomson C. Tumour suppressors and cell metabolism: a recipe for cancer growth. Genes Dev 2009; 23: 537-548.

141 Skala MC, Riching KM, Gendron-Fitzpatrick A, et al. In vivo multiphoton microscopy of NADH and FAD redox states, fluorescence lifetimes, and cellular morphology in precancerous epithelia. Proc Natl Acad Sci 2007; 104: 19494-19499.

142 Awasthi K, Chang F-L, Hsieh P-Y, et al. Characterization of endogenous fluorophores in nonsmall lung cancerous cells: a comparison with nonmalignant lung normal cells. J Biophotonics 2020; 13: e201960210.

143 Blacker TS, Mann ZF, Gale JE, et al. Separating NADH and NADPH fluorescence in live cells and tissues using FLIM. Nat Commun 2014; 5: 3936. 
Wang Y, Song C, Wang M, et al. Rapid, label-free, and highly sensitive detection of cervical cancer with fluorescence lifetime imaging microscopy. IEEE J Sel Top Quantum Electron 2016; 22: 228-234.

145 Cheng S, Yon Hwang D, Cuenca R, et al. In vivo detection of oral epithelial pre-cancer and cancer by endogenous fluorescence lifetime imaging (FLIM) endoscopy. Biomed Opt 2016: CTh4A.3.

146 Riemann I, Ehlers A, Le Harzic R, et al. Non-invasive analysis/diagnosis of human normal and melanoma tissues with two-photon FLIM in vivo. Proc SPIE 2008; 6842: 684205.

147 Kantelhardt S, Kalasauskas D, Konig K, et al. In vivo multiphoton tomography and fluorescence lifetime imaging of human brain tissue. J Neurooncol 2016; 127: 473-482.

148 Kennedy GT, Coda S, Thompson AJ, et al. Fluorescence lifetime imaging endoscopy. Proc SPIE 2011; 7893: 789308.

149 Downes A, Elfick A. Raman spectroscopy and related techniques in biomedicine. Sensors 2010; 10: 1871-1889.

150 Huang Z, McWilliams A, Lui H, et al. Near-infrared Raman spectroscopy for optical diagnosis of lung cancer. Int J Cancer 2003; 107: 1047-1052.

151 Short MA, Lam S, McWilliams A, et al. Using laser Raman spectroscopy to reduce false positives of autofluorescence bronchoscopies: a pilot study. J Thorac Oncol 2011; 6: 1206-1214.

152 Mahadevan-Jansen A, Richards-Kortum R. Raman spectroscopy for the detection of cancers and precancers. J Biomed Opt 1996; 1: 31-70.

153 Zheng Q, Li J, Yang L, et al. Raman spectroscopy as a potential diagnostic tool to analyse biochemical alterations in lung cancer. Analyst 2020; 145: 385-392.

154 Keller MD, Kanter EM, Mahadevan-Jansen A. Raman spectroscopy for cancer diagnosis. Spectroscopy 2006; 21: 33-41.

155 McGregor HC, Short MA, Lam S, et al. Development and in vivo test of a miniature Raman probe for early cancer detection in the peripheral lung. J Biophotonics 2018; 11: e201800055.

156 Dochow S, Ma D, Latka I, et al. Combined fiber probe for fluorescence lifetime and Raman spectroscopy. Anal Bioanal Chem 2015; 407: 8291-8301.

157 Amitonova LV, de Boer JF. Compressive endo-microscopy. Proc SPIE 2019; 11076: 110760J. 